\title{
FORCING AND MODELS OF ARITHMETIC ${ }^{1}$
}

\author{
S. G. SIMPSON
}

ABSTRACr. It is shown that every countable model of Peano arithmetic with finitely many extra predicates (or of ZFC with finitely many extra predicates) is a reduct of a pointwise definable such model.

This note applies the forcing method to a question concerning definability in models of Peano arithmetic.

THEOREM. Let $M=\langle|M|,+, \cdot\rangle$ be a countable model of Peano arithmetic. ${ }^{2}$ Then there is a set $U \subseteq|M|$ such that

(i) $\langle M, U\rangle$ satisfies the first order induction schema for formulas containing an extra predicate $\boldsymbol{U}(x)$;

(ii) every element of $|M|$ is first order definable in $\langle M, U\rangle$.

Proof. A condition is an $M$-finite sequence of 0 's and 1's, i.e. a mapping $p:\left\{b \mid b<{ }^{M} a\right\} \rightarrow\{0,1\}$ such that $a \in|M|$ and $p$ is coded by an element of $|M|$. We use $p, q, \cdots$ as variables ranging over conditions. A set of conditions is dense if every condition is extended by some condition in the set. Let $\left\langle a_{n} \mid n<\omega\right\rangle$ enumerate the elements of $|M|$. Let $\left\langle D_{n} \mid n<\omega\right\rangle$ enumerate the dense sets of conditions which are first order definable over $M$ allowing parameters from $|M|$. It is safe to assume:

the parameters in the first order definition of $D_{n}$ are among $a_{0}, a_{1}, \cdots, a_{n-1}$.

Define a sequence of conditions $\left\langle p_{n}\right| n\langle\omega\rangle$ by $p_{0}=\varnothing ; p_{2 n+1}=$ the $<^{M^{-}}$ least condition $q \supseteq p_{2 n}$ such that $q \in D_{n} ; p_{2 n+2}=p_{2 n+1}$ followed by a string of $a_{n} 0$ 's followed by a 1 . Define $U \subseteq|M|$ by letting $\bigcup\left\{p_{n} \mid n<\omega\right\}$ be the characteristic function of $U$. To prove that $\langle M, U\rangle$ satisfies first order induction, use the genericity of $U$.

[Details. Let $L$ be the first order language with $+, \cdot, \boldsymbol{U}(x)$, and constant symbols $a$ for each $a \in|M|$. For $\theta$ a sentence of $L$ define the (strong)

Received by the editors May 16, 1973.

AMS (MOS) subject classifications (1970). Primary 02H05, 02H13, 02H20.

1 This research was partially supported by NSF contract GP-24352.

${ }^{2}$ Peano arithmetic is the theory $\mathbf{P}$ of Shoenfield, Mathematical logic, AddisonWesley, 1967.

(c) American Mathematical Society 1974 
forcing relation $p \Vdash \theta$ by

$$
\begin{array}{lll}
p \Vdash \boldsymbol{a}+\boldsymbol{b}=\boldsymbol{c} & \text { iff } & \boldsymbol{a}+\boldsymbol{b}=c ; \\
p \Vdash \boldsymbol{a} \cdot \boldsymbol{b}=\boldsymbol{c} & \text { iff } & a \cdot b=c ; \\
p \Vdash \boldsymbol{U}(\boldsymbol{a}) & \text { iff } & p(a)=1 ; \\
p \Vdash \theta_{1} \vee \theta_{2} & \text { iff } & p \Vdash \theta_{1} \text { or } p \Vdash \theta_{2} ; \\
p \Vdash 7 \theta & \text { iff } & q \Vdash \theta \text { for no } q \supseteq p ; \\
p \Vdash \exists x \theta(x) & \text { iff } & p \Vdash \theta(\boldsymbol{a}) \text { for some } a \in|M| .
\end{array}
$$

Prove the basic forcing lemmas as usual. It remains to show that $\varnothing \Vdash 77(\exists x \theta(x) \rightarrow \exists$ least $x$ such that $\theta(x))$.

Suppose $p \Vdash \exists x \theta(x)$. Then $p \Vdash \theta(a)$ for some $a \in|M|$. Working within $M$, define a sequence of conditions $\left\langle q_{c} \mid c<^{M} b+1\right\rangle$ where $b<^{M} a+1$ as follows: $q_{0}=p ; q_{c+1}=$ the $<^{M}$-least $q \supseteq q_{c}$ such that $q \Vdash 7 \theta(c) ; b=$ the $<^{M}$-least $c$ such that $q_{c+1}$ is undefined. Then $q_{b} \supseteq p$ and $q_{b} \Vdash 77(b$ is the least $x$ such that $\theta(x))$.]

On the other hand, using (*), one easily shows by induction on $n$ that $p_{2 n+1}, p_{2 n+2}$, and $a_{n}$ are first-order definable in $\langle M, U\rangle$. This completes the proof.

REMARK. One can apply the same method to models of set theory to get the following theorem: Let $M=\left\langle|M|, \epsilon^{M}\right\rangle$ be a countable model of $Z F C$, then there is a set $U \subseteq|M|$ such that

(i) $\langle M, U\rangle$ satisfies the first-order replacement schema for formulas containing an extra predicate $\boldsymbol{U}(x)$;

(ii) every element of $|M|$ is first-order definable in $\langle M, U\rangle$.

This is an improvement of the theorem of U. Felgner, Fund. Math. 71 (1971), 43-62.

Department of Mathematics, University of California, Berkeley, California 94720 